\title{
Generation and Processing of Complex Photon States with Quantum Frequency Combs
}

\author{
Stefania Sciara, Piotr Roztocki, Cristina Rimoldi, Mario Chemnitz, Bennet Fischer, Luis Romero \\ Cortés, William J. Munro, David J. Moss, Fellow, IEEE, Lucia Caspani, Christian Reimer, José \\ Azaña, Michael Kues, and Roberto Morandotti, Senior Member, IEEE
}

\begin{abstract}
The development of quantum technologies for quantum information (QI) science demands the realization and precise control of complex (multipartite and high dimensional) entangled systems on practical and scalable platforms. Quantum frequency combs (QFCs) represent a powerful tool towards this goal. They enable the generation of complex photon states within a single spatial mode as well as their manipulation using standard fiber-based telecommunication components. Here, we review recent progress in the development of QFCs, with a focus on results that highlight their importance for the realization of complex quantum states. In particular, we outline recent work on the use of integrated QFCs for the generation of high-dimensional multipartite optical cluster states - lying at the basis of measurement-based quantum computation. These results confirm that the QFC approach can provide a stable, practical, low-cost, and established platform for the development of quantum technologies, paving the way towards the advancement of QI science for out-of-the-lab applications, ranging from practical quantum computing to more secure communications.
\end{abstract}

Index Terms - coherent control of photon states, computing and information science, fiber-based telecommunications, highdimensional multipartite entanglement, photon cluster states, photonic integrated circuits, practical and scalable quantum technology, quantum frequency combs.

\section{INTRODUCTION}

$\mathrm{T}$ HE generation and processing of complex quantum states offer significant and promising advantages for various tasks, such as quantum communications [1], computing [2], sensing [3], and metrology [4]. Two approaches can be considered to realize such states: one makes use of multiple two-level systems (i.e., qubits), while the other consists in increasing the number of levels per single system (i.e., using qudits). Multipartite qubit states are typically achieved through

S. Sciara, P. Roztocki, C. Rimoldi, M. Chemnitz, L. Romero Cortés, J. Azaña are with the Centre Énergie Matériaux Télécommunications, Institut National de la Recherche Scientifique, Varennes, QC J3X 1S2, Canada (emails: stefania.sciara@emt.inrs.ca;

cristina.rimoldi@emt.inrs.ca; piotr.roztocki@emt.inrs.ca; mario.chemnitz@emt.inrs.ca;

W.J. Munro is with NTT Basic Research Laboratories, NTT Corporation, Kanagawa, Japan (email: bilmun@qis1.ex.nii.ac.jp).

D.J. Moss is with the Centre for Microphotonics, Swinburne University of Technology, Hawthorn, Vic.3122, Australia (email: dmoss@swin.edu.au). platforms such as trapped ions [5], superconducting circuits [6], and quantum dots [7]. Their scaling is however difficult, mainly due to the interactions with both neighboring particles and the environment. In sharp contrast, photons, i.e. the quantum states of light, have been shown to be ideally suited for practical realization of qudits, as they allow for significant information encoding in their several degrees of freedom (DOFs), such as time and frequency [8]-[12], or orbital angular momentum [13]. Furthermore, due to the compatibility with off-the-shelf telecommunication components, photonic systems pave the way towards a robust qudit transmission platform. In this context, QFCs have allowed for a significant reduction in the system footprint. Frequency combs are light sources with a spectrum consisting of a series of discrete, equally-spaced spectral modes. In the classical domain, they have made possible the interfacing of optical and microwave frequency regimes, with consequent important innovations for spectroscopy and metrology [14]. Allowing for entanglement generation over many frequency modes in a single spatial mode [15], QFCs have been exploited for the realization of highdimensional photon states [11], [16]. First implementations of QFCs were achieved through parametric down-conversion in free-space optical parametric oscillators [17]-[19], leading to the generation of so-called squeezed states in a continuousvariable $(\mathrm{CV})$ formalism, where the DOFs are given by the electromagnetic field quadratures. $\mathrm{CV}$ quantum states have been very recently demonstrated in integrated photonic platforms [20]. However, getting access to the individual frequency modes for quantum state control can be challenging [21]. The recent use of integrated technology for the generation of photon states in discrete (i.e., qudit) frequency modes has simplified the control of the single spectral modes. This can dramatically increase the scalability, as well as lower the cost of the setups used for QFC generation [22], [23]. This review

L. Caspani is with the University of Strathclyde, Glasgow G1 1RD, UK (email: lucia.caspani@strath.ac.uk).

C. Reimer is with HyperLight Corporation, Cambridge, USA (email: christian@hyperlightcorp.com).

M. Kues is with Leibnitz University Hannover, 30167 Hannover, Germany (e-mail: michael.kues@hot-uni.hannover.de).

R. Morandotti is with the Centre Énergie Matériaux Télécommunications, Institut National de la Recherche Scientifique, Varennes, QC J3X 1S2, Canada.

$\mathrm{He}$ is also an adjoint faculty with University of Electronic Science and Technology of China, 610054 Chengdu, China and with ITMO University, 197101 St. Petersburg, Russia (email: morandotti@emt.inrs.ca). 
focuses specifically on the use of QFCs for the generation of complex states of qudits, as well as on their promising potential for quantum telecommunications and computing applications.

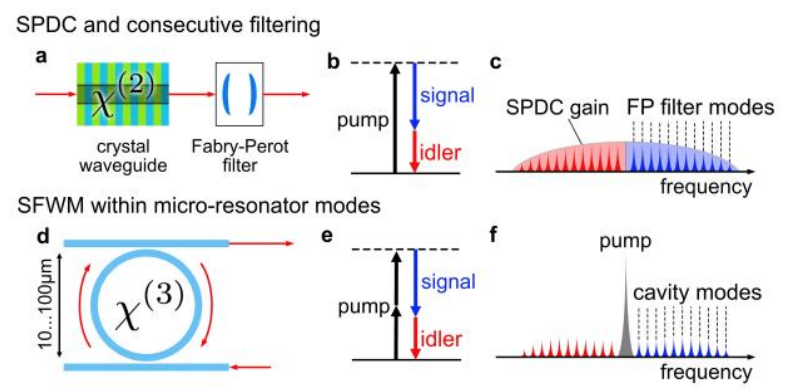

Fig. 1. Principles of entangled photon pairs generation using (a-c) SPDC and (d-f) SFWM: (a, d) experimental setup schemes, (b, e) energy conservation graph, (c, f) output mode spectrum of the QFCs. The generated photon pair is entangled across the frequency bins.

\section{GENERATION OF QUANTUM FREQUENCY COMBS}

Many configurations exist for realizing QFCs. In Fig. 1 we represent the two commonly used ones. The first relies on the generation of entangled photon pairs over a broad bandwidth using spontaneous parametric down-conversion (SPDC) in periodically poled crystal waveguides (e.g., PPLN [24]) with subsequent frequency filtering. While this approach benefits from high generation efficiencies, the narrow spectral filtering inevitably reduces the high photon flux of SPDC. The second approach relies on embedding the nonlinear medium inside an optical cavity, so photon pairs are directly generated at the required frequencies [25]. Configurations including integrated SPDC in a cavity has been successfully achieved very recently [26], but on-chip integration becomes easier for third-order nonlinear media exploiting spontaneous four-wave mixing (SFWM) directly in equally-spaced frequency modes within integrated micro-resonators [9], [11], [15], [16], [22], [27][34]. As compared to the configuration that includes SFWM with filtering, this approach benefits from the coherent field enhancement of a low-power excitation source, which overcomes the weaker generation efficiency of the SFWM process [22].

Both approaches allow to generate signal and idler photons that, obeying energy conservation laws, are entangled over multiple well-defined frequency modes, as demonstrated via quantum interference and Bell inequality violations employing electro-optical modulation and frequency filtering [10], [11].

These techniques enable the efficient generation of QFCs with well-defined entanglement properties, readily usable for significant applications in QI technologies. As a relevant example of such a realization, we will discuss the generation and processing of high-dimensional cluster states [34].

\section{GENERATION OF OPTICAL QUDIT CLUSTER STATES}

Cluster states [35] are a class of multipartite states with unique entanglement properties (maximal connectedness and highest persistency of entanglement), which make them fundamental for the realization of one-way quantum computers, where the computational resource is provided in the physical form of a cluster state, while the actual computation consists of single-party projection measurements [36], [37]. Optical cluster states can be generated either in the $\mathrm{CV}$ or in the discretevariable (DV) formalism. CV cluster states [20], [38], [39], can be achieved using squeezed photons and are intrinsically highdimensional (e.g., a sixteen spectral mode CV cluster state was demonstrated in [38]). However, the level of squeezing can be very sensitive to noise. DV cluster states, despite the loss associated with integrated platforms, are more noise robust. However, their experimental realization was limited to qubits [37], [40]-[43], and only entangled DOFs such as polarization [40], [44] and linear momentum [42] have been exploited for their demonstration. Increasing the number of photons to boost the computational resource comes at the price of reduced detection rates and robustness to noise [41]. As a result, the realization of cluster states has been limited to eight qubits [44]. This problem can be addressed by using qudits, which provide a more powerful computational resource, as well as a higher noise tolerance [11], [16], [45]-[47]. Here, we describe the first demonstration of a DV qudit multipartite cluster state [16].

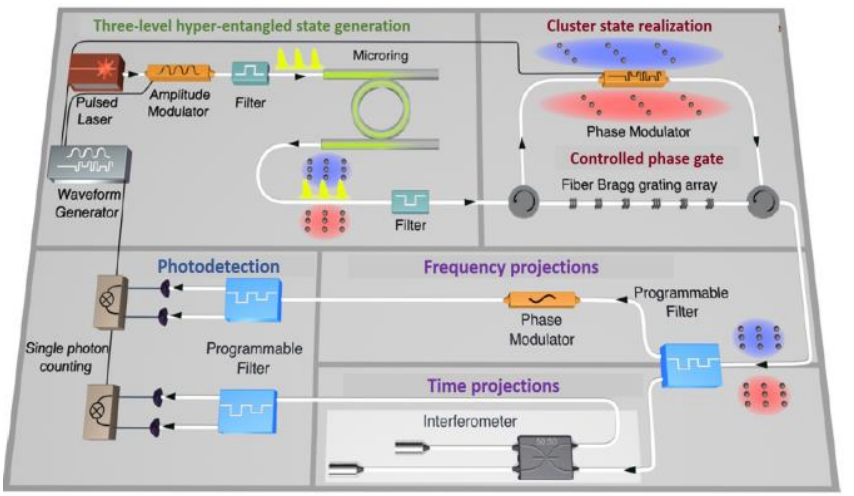

Fig. 2. Triple pulses generated through a mode-locked laser excite the resonance frequency of a microring resonator. Here, signal (red) and idler (blue) photons are generated via SFWM, both in a superposition of three time and three frequency modes (indicated by bullets), thus forming a hyper-entangled state. A controlled phase gate was employed for realizing the cluster state. This was then sent through a programmable filter into two different analysis setups: 1) an electro-optic phase modulator for frequencybin projections and 2) an unbalanced interferometer for time-bin projections. Finally, the photons were separated by filters and measured with single-photon detectors. Adapted from [16].

The basis for such a realization was a two-photon state generated through SFWM over a QFC covering the telecom Cband [9] and exhibiting simultaneous entanglement (i.e. hyperentanglement) [48], [49] in time [8], [9] and frequency [10], [11], which can be treated as independent DOFs since the timebandwidth product is much larger than 1 (which is the limit for Einstein-Podolsky-Rosen quantum correlations) [16]. Time entanglement was created by exciting a nonlinear microring resonator [9] with three consecutive mode-locked pulses, while frequency entanglement was generated simultaneously by selecting three microring resonances per photon (see Fig. 2). In order to transform such entangled-product state into a cluster state, it was necessary to get access to its individual frequency components and add specific phases. For this end, a controlled 
phase gate was employed for temporally dispersing each frequency mode into different time slots (i.e., frequency-to-time mapping). This specific task was implemented through a fiber Bragg grating array, while the phase terms were changed with a temporally synchronized phase modulator (see Fig. 2). By choosing the appropriate phase pattern and by coherently reversing the frequency-to-time mapping, the output of the phase gate resulted in a four-partite three-level cluster state (see Fig. 2) [16].
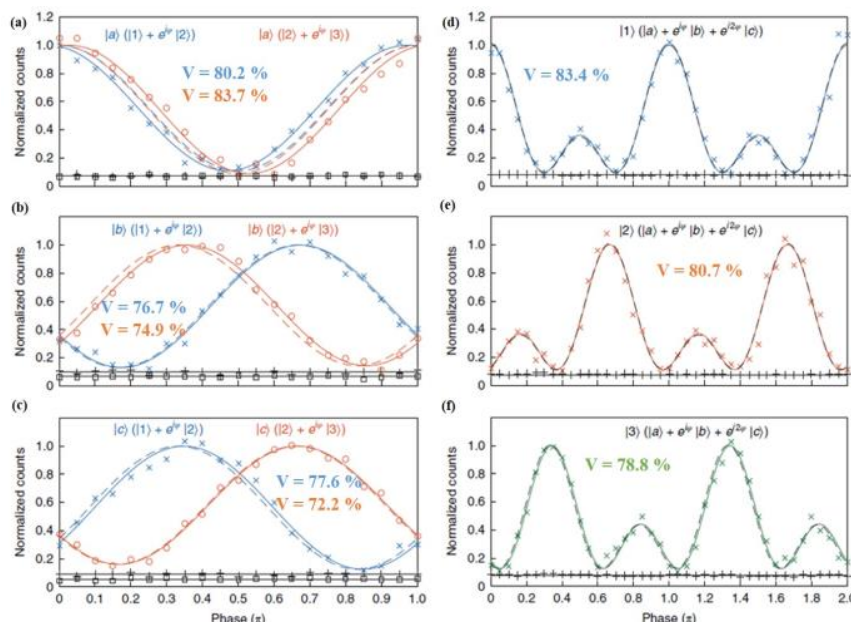

Fig. 3. Quantum interference measurements in (a-c) time and (df) frequency domains to confirm the entanglement of the projected two-partite states. The quantum interference follows the expected functions for the ideal two-partite entangled states (dashed line: theory, solid line: fit). The extracted visibilities exceeded their respective thresholds (i.e. $70.7 \%$ in a-c, and $77.4 \%$ in d-f), thus violating the respective Bell inequalities. Adapted from [16].

The cluster state realization was validated by developing a dlevel entanglement witness [45]. A negative measured expectation value of $-0.28 \pm 0.04$ confirmed that the demonstrated state exhibited genuine four-partite three-level entanglement [16]. Finally, the cluster state was used to implement proof-of-principle high-dimensional one-way quantum processing, consisting here in projecting any qudit pair of the cluster state either in time or frequency. Time and frequency projections were performed in fiber with a two-arm interferometer and a phase modulator, respectively (see Fig. 2). By implementing these measurements, the remaining qudits were projected into mutually orthogonal bipartite entangled states, which is the working principle of one-way quantum computers [36]. The entanglement and the orthogonality of the two-partite states were validated through quantum interference measurements and Bell's inequality violations (see Fig. 3) [16].

\section{APPLICATIONS IN QUANTUM STATE PROCESSING AND TELECOMMUNICATIONS}

Qudit systems have been foreseen to outperform their qubit counterpart in multiple applications for quantum computing and telecommunications. The access to many information channels (such as frequency modes) promises a significant reduction of the system complexity [50], and an increase of both noise robustness and acceptable error rates of quantum communication links [51]. Recent years have seen a growing number of proof-of-concept applications of QFCs demonstrating their performance for quantum technologies.

To be useful for quantum computing, photon qudits require the implementation of generalized Pauli ( $X$ and $Z$ ) and Hadamard $(H)$ gates in order to process the information content of the whole quantum state. In the telecom C-band regime, an $H$ gate was demonstrated by using a programmable filter placed between two electro-optical modulators, enabling qubit and qutrit control across up to three frequency modes with nearunity fidelity [52]. In principle, a proper combination of $H$ and $Z$ gates can be further used to implement the $X$ gate, thus completing the set of universal gates in the frequency domain. However, from a practical viewpoint, the individual losses per gate prevent a cascaded application of the demonstrated architectures, while loss engineering or alternative schemes remain key problems to be solved. Very recently, a generalized $\mathrm{X}$ gate was demonstrated in the temporal domain, which provided the base for a generalized controlled-NOT (i.e. SUM) gate operating on selected qubits in a 32-dimensional timefrequency state space [53]. Such different, yet noncomplementary, sets of universal quantum gates thus bring photon-based computation within practical reach.

Significant advances in the use of QFCs were also achieved in quantum telecommunications. In quantum key distribution, which guarantees for secure message transmissions, protocols making use of the increased information capacity per qudit have been proposed for time-bin entangled photons [54] and tested with up to 8.7 key bits per coincidence [55] and a secure key rate of up to $26 \mathrm{Mbit} / \mathrm{s}$ [56]. Similar achievements can be anticipated from frequency encoding [57], well established in classical telecom networks. Further protocols rely on, e.g., quantum repeaters [58], which build on the generation and recurrent recreation of an interconnected entanglement network between multiple parties of a so-called repeater graph state (e.g., cluster state) in order to transfer a quantum key. First experimental realizations have been reported by processing 6 independent photon pairs [59], which would clearly benefit from the increased information content of qudit states.

\section{CONCLUSIONS}

Integrated QFCs have been shown a valid, scalable, and versatile platform for the generation of complex quantum states. They further allow for state manipulation within a single spatial mode via off-the-shelf telecommunication components. This makes QFCs a highly promising platform for optical QI processing. In the reviewed work, QFCs have been exploited for the first demonstration of a DV qudit optical cluster state. The results presented here pave the way for the use of such cluster state towards one-way quantum computing and as a basis for information-dense quantum key distribution via frequency encoding, or quantum repeaters. Making use of fewphoton qudit states has the potential to boost computing power and performance [16], [50], due to noise robustness and reduction of system complexity. Finally, the possibility to implement gates and algorithms in scalable and telecomcompatible platforms is promising for making quantum computers market-accessible in the near future. 


\section{REFERENCES}

[1] H. J. Kimble, "The quantum internet," Nature, vol. 453, no. 7198, pp. 1023-1030, 2008.

[2] T. D. Ladd et al., "Quantum computers," Nature, vol. 464, no. 7285, pp. 45-53, Mar. 2010.

[3] S. Lloyd, "Enhanced sensitivity of photodetection via quantum illumination," Science, vol. 321, no. 5895, pp. 1463-1465, Sep. 2008.

[4] V. Giovannetti et al., "Advances in quantum metrology," Nat. Photonics, vol. 5, no. 4, pp. 222-229, 2011.

[5] R. Blatt et al., "Entangled states of trapped atomic ions," Nature, vol. 453, no. 7198, pp. 1008-1015, 2008.

[6] M. H. Devoret et al., "Superconducting circuits for quantum information: an outlook," Science, vol. 339, pp. 1169-1174, 2013.

[7] E. V. Denning et al., "Protocol for generating multiphoton entangled states from quantum dots in the presence of nuclear spin fluctuations," Phys. Rev. A, vol. 96, no. 6, pp. 1-9, 2017.

[8] J. Brendel et al., "Pulsed energy-time entangled twin-photon source for quantum communication," Phys. Rev. Lett., vol. 82, no. 12, pp. 2594--2597, 1999.

[9] C. Reimer et al., "Generation of multiphoton entangled quantum states by means of integrated frequency combs," Science, vol. 351, no. 6278 , pp. 1176-1180, 2016.

[10] L. Olislager et al., "Frequency-bin entangled photons," Phys. Rev. A - At. Mol. Opt. Phys., vol. 82, no. 1, pp. 1-7, 2010.

[11] M. Kues et al., "On-chip generation of high-dimensional entangled quantum states and their coherent control," Nature, vol. 546, no. 7660, pp. 622-626, 2017.

[12] B. Brecht et al., "Photon temporal modes: a complete framework for quantum information science," Phys. Rev. X, vol. 5, no. 4, p. 041017, 2015.

[13] A. Mair et al., "Entanglement of the orbital angular momentum states of photons," Nature, vol. 412, no. 6844, pp. 313-316, Jul. 2001.

[14] A. Schliesser et al., "Mid-infrared frequency combs," Nat. Photonics, vol. 6, no. 7, pp. 440-449, 2012.

[15] L. Caspani et al., "Multifrequency sources of quantum correlated photon pairs on-chip: a path toward integrated quantum frequency combs," Nanophotonics, vol. 5, no. 2, pp. 351-362, 2016.

[16] C. Reimer et al., "High-dimensional one-way quantum processing implemented on d-level cluster states," Nat. Phys., vol. 15, no. 2, pp. 148-153, 2019.

[17] M. Pysher et al., "Parallel generation of quadripartite cluster entanglement in the optical frequency comb," Phys. Rev. Lett., vol. 107, no. 3, pp. 2-5, 2011.

[18] J. Roslund et al., "Wavelength-multiplexed quantum networks with ultrafast frequency combs," Nat. Photonics, vol. 8, no. 2, pp. 109112, Feb. 2014.

[19] Y. J. Lu et al., "Mode-locked two-photon states," Phys. Rev. Lett., vol. 91, no. 16, pp. 1636021-1636024, Oct. 2003.

[20] F. Lenzini et al., "Integrated photonic platform for quantum information with continuous variables," Sci. $A d v$., vol. 4, no. 12, pp. $1-8,2018$.

[21] M. Chen et al., "Experimental realization of multipartite entanglement of 60 Modes of a quantum optical frequency comb," Phys. Rev. Lett., vol. 112, no. 12, p. 120505, Mar. 2014.

[22] P. Roztocki et al., "Complex quantum state generation and coherent control based on integrated frequency combs," J. Light. Technol., vol. 37, no. 2, pp. 338-344, Jan. 2019.

[23] M. Kues et al., "Quantum optical microcombs," Nature Photonics, vol. 13, no. 3. Nature Publishing Group, pp. 170-179, 01-Mar-2019.

[24] H.-H. Lu et al., "Quantum interference and correlation control of frequency-bin qubits," Optica, vol. 5, no. 11, p. 1455, Nov. 2018.

[25] E. Pomaríco et al., "Waveguide-based OPO source of entangled photon pairs," New J. Phys., vol. 11, 2009.

[26] M. Stefszky et al., "Towards optical-frequency-comb generation in continuous-wave-pumped titanium-indiffused lithium-niobate waveguide resonators," Phys. Rev. A, vol. 98, no. 5, pp. 1-9, 2018.

[27] J. A. Jaramillo-Villegas et al., "Persistent energy-time entanglement covering multiple resonances of an on-chip biphoton frequency comb," Optica, vol. 4, no. 6, p. 655, 2017.

[28] R. Kumar et al., "Spectrally multiplexed and tunable-wavelength photon pairs at $155 \mu \mathrm{m}$ from a silicon coupled-resonator optical waveguide," Opt. Lett., vol. 38, no. 16, p. 2969, 2013.

[29] W. C. Jiang et al., "Silicon-chip source of bright photon pairs," Opt. Express, vol. 23, no. 16, p. 20884, 2015.
[30]

D. J. Moss et al., "New CMOS-compatible platforms based on silicon nitride and Hydex for nonlinear optics," Nat. Photonics, vol. 7, no. 8, pp. 597-607, 2013.

[31] P. Roztocki et al., "Practical system for the generation of pulsed quantum frequency combs," Opt. Express, vol. 25, no. 16, p. 18940, 2017.

[32] L. Caspani et al., "Integrated sources of photon quantum states based on nonlinear optics," Light Sci. Appl., vol. 6, no. 11, p. e17100, Nov. 2017.

[33] A. Pasquazi et al., "Micro-combs: a novel generation of optical sources," Phys. Rep., vol. 729, pp. 1-81, 2018.

[34] P. Imany et al., "50-GHz-spaced comb of high-dimensional frequency-bin entangled photons from an on-chip silicon nitride microresonator," Opt. Express, vol. 26, no. 2, p. 1825, Jan. 2018.

[35] H. J. Briegel et al., "Persistent entanglement in arrays of interacting particles," Phys. Rev. Lett., vol. 86, no. 5, pp. 910-913, Jan. 2001.

[36] R. Raussendorf et al., "A one-way quantum computer," Phys. Rev. Lett., vol. 86, no. 22, pp. 5188-5191, May 2001.

[37] P. Walther et al., "Experimental one-way quantum computing," Nature, vol. 434, no. 7030, pp. 169-176, 2005.

[38] Y. Cai et al., "Multimode entanglement in reconfigurable graph states using optical frequency combs," Nat. Commun., vol. 8, no. 1, p. 15645, Aug. 2017.

[39] J. Nokkala et al., "Reconfigurable optical implementation of quantum complex networks," New J. Phys., vol. 20, no. 5, 2018.

[40] K. Chen et al., "Experimental realization of one-way quantum computing with two-photon four-qubit cluster states," Phys. Rev. Lett., vol. 99, no. 12, pp. 1-4, 2007.

[41] C. Y. Lu et al., "Experimental entanglement of six photons in graph states," Nat. Phys., vol. 3, no. 2, pp. 91-95, 2007.

[42] G. Vallone et al., "Realization and characterization of a two-photon four-qubit linear cluster state," Phys. Rev. Lett., vol. 98, no. 18, p. $180502,2007$.

[43] G. Vallone et al., "Active one-way quantum computation with twophoton four-qubit cluster states," Phys. Rev. Lett., vol. 100, no. 16, pp. 2-5, 2008.

[44] X.-C. Yao et al., "Experimental demonstration of topological error correction," Nature, vol. 482, no. 7386, pp. 489-494, Feb. 2012.

[45] S. Sciara et al., "Universal N-partite d-level pure-state entanglement witness based on realistic measurement settings," Phys. Rev. Lett., vol. 122, no. 12, p. 120501, 2019.

[46] D. Collins et al., "Bell inequalities for arbitrarily high-dimensional systems," Phys. Rev. Lett., vol. 88, no. 4, p. 4, 2002.

[47] A. C. Dada et al., "Experimental high-dimensional two-photon entanglement and violations of generalized Bell inequalities," Nat. Phys., vol. 7, no. 9, pp. 677-680, 2011.

[48] P. G. Kwiat, "Hyper-entangled states," vol. 0340, no. 1997, 2009.

[49] J. T. Barreiro et al., "Generation of hyperentangled photon pairs," Phys. Rev. Lett., vol. 95, no. 26, pp. 1-4, 2005.

[50] B. P. Lanyon et al., "Simplifying quantum logic using higherdimensional Hilbert spaces," Nat. Phys., vol. 5, no. 2, pp. 134-140, Feb. 2009.

[51] N. J. Cerf et al., "Security of quantum key distribution using d-level systems," Phys. Rev. Lett., vol. 88, no. 12, p. 127902, Mar. 2002.

[52] H.-H. Lu et al., "Electro-optic frequency beam splitters and tritters for high-fidelity photonic quantum information processing," Phys. Rev. Lett., vol. 120, no. 3, p. 030502, Jan. 2018.

[53] P. Imany et al., "High-dimensional optical quantum logic in large operational spaces," npj Quantum Inf., vol. 5, no. 1, p. 59, Dec. 2019.

[54] I. Ali-Khan et al., "Large-alphabet quantum key distribution using energy-time entangled bipartite states," Phys. Rev. Lett., vol. 98, no. 6, 2007.

[55] T. Zhong et al., "Photon-efficient quantum key distribution using time-energy entanglement with high-dimensional encoding," New J. Phys., vol. 17, 2015.

[56] N. T. Islam et al., "Provably secure and high-rate quantum key distribution with time-bin qudits," $S c i$. $A d v$., vol. 3, no. 11, pp. 1-7, 2017.

[57] S. Wengerowsky et al., "An entanglement-based wavelengthmultiplexed quantum communication network," Nature, vol. 564, no. 7735, pp. 225-228, Dec. 2018.

[58] K. Azuma et al., "All-photonic quantum repeaters," Nat. Commun., vol. 6, pp. 1-7, 2015.

[59] Z.-D. Li et al., "Experimental demonstration of all-photonic quantum repeater," Conf. Lasers Electro-Optics, p. FTh4A.6, 2019. 